\begin{tabular}{lrr} 
STUDIA & ROMANICA & POSNANIENSIA \\
\hline UAM & Vol. $41 / 1$ & Poznań 2014
\end{tabular}

SYLWIA MIKOLAJCZAK

Universidad Adam Mickiewicz de Poznań

\title{
ASPECTOS POSITIVOS DE LAS INFLUENCIAS INTRALINGÜÍSTICAS EN EL PROCESO DE ADQUISICIÓN DE L2
}

\begin{abstract}
Sylwia Mikołajczak, Aspectos positivos de las influencias intralingüisticas en el proceso de adquisición de $L 2$ [Positive aspects of interlinguistic influences in the process of L2 acquisition], Studia Romanica Posnaniensia, Adam Mickiewicz University Press, Poznań, vol. XLI/1: 2014, pp. 119-127. ISBN 978-83-232-2673-4. ISSN 0137-2475. eISSN 2084-4158. Doi: 10.7169/strop2014.411.008

The processes that take place in the mind of a multilingual person during the second language acquisition are mostly shrouded in mystery. We can only observe traces of these processes manifesting themselves in the interpenetration of two or more linguistic systems. The current study aims to recognize how the knowledge of L1, in this case Spanish, influences on the acquisition and usage of the target language Portuguese (L2), and how to take advantage of the interferences to make the L2 learners succeed. The possibility of linguistic influences grows with each acquired language. There are also important contributing factors such as a degree of similarity between the languages, a level of proficiency in each of them, and the manner and time of a language acquisition. During the Portuguese course at the Spanish Philology, we can observe such phenomena as: code switching or total displacement, hybrids, false friends, multi-word units calquing, and morphogrammatical transfer. Although one of the factors that affects the L2 acquisition is the level of proficiency, the students hardly take advantage of their mother tongue, selecting the language typologically closer to Portuguese, Spanish. The students use the previously acquired knowledge to create analogies that should be considered as an intermediate step in the acquisition of L2, not as something negative, but rather a means for providing a starting point for the analysis of error which, consequently, leads to improvement.
\end{abstract}

Keywords: language transfer, interlinguistic influences, language acquisition, language interferences, interlanguage

\section{INTRODUCCIÓN. DEFINICIÓN DE TRANSFERENCIA, INFLUENCIAS LINGÜÍSTICAS E INTERFERENCIAS}

Los procesos que ocurren en la mente de una persona políglota a la hora de crear un enunciado en una lengua meta están, en su mayoría, llenos de incógnitas sin aclarar. Solo podemos observar las huellas de dichos procesos en los elementos pertenecientes a dos o más sistemas lingüísticos, puesto que durante la producción se llega a activar el conocimiento de otros idiomas almacenados en el cerebro en la memoria de larga duración. En este contexto, el objetivo de la presente investigación es, pues, doble: a) indagar cómo el conocimiento del idioma L1, en este caso el español, repercute en la 
adquisición y uso del idioma básico (el portugués L2), y b) estudiar cómo aprovechar las interferencias para que influyan positivamente en el aprendizaje de L2.

Aunque algunos autores, como Paradis (2004), afirman que las influencias lingüísticas son el resultado de mecanismos neurobiológicos, parecidos a los que ocurren en la mente de una persona monolingüe en una situación de cambio de registro lingüístico, sin embargo, la mayoría de autores opina que las influencias lingüísticas de las personas que hablan tres o más idiomas son de un mayor grado de complejidad, apreciándose una calidad y cantidad más desarrollada (Cenoz, 2001). Si bien el número de posibles influencias lingüísticas aumenta a medida que se adquiere un idioma, también intervienen otros factores, como el grado de parentesco entre las lenguas, el nivel de competencia en cada una de ellas, el modo como se adquiere y el tiempo destinado a su aprendizaje.

La idea de las influencias lingüísticas (en inglés: cross-linguistic influence) fue introducida en los años 80 del siglo XX por los lingüistas Eric Kellerman y Michael Sharwood Smith (Chłopek, 2011: 141), cuando examinaban las relaciones intralingüísticas reales, sin fijarse únicamente, como sus antecesores, en la transferencia negativa en el ámbito de la didáctica del idioma extranjero (behavioristas) o en los aspectos sociolingüísticos (voces de procedencias extranjeras y cambios de códigos) descritos en los ambientes bilingües. Las influencias lingüísticas que incluyen en sí mismas ideas como «transferencia, interferencias, evitación, voz de procedencia extranjera y aspectos de la pérdida del idioma» (trad. nuestra de Chłopek, 2011: 141), se entienden actualmente como «la influencia de cada tipo (por lo menos) de una lengua (o interlengua) en otra lengua (o interlengua) cualquiera» (trad. nuestra, Chłopek, 2011: 141). Dichas influencias pueden tener carácter no intencional, si se aprende a usar de una manera inconsciente un idioma diferente al idioma básico, o intencional, si se aprende conscientemente. El resultado de dichas influencias puede ser considerado aceptable o inaceptable (es decir, erróneo). Entre las influencias intralingüísticas se pueden incluir:

- la transferencia lingüística (language transfer, Odlin, 1989)1,

- las voces de procedencia extranjera,

- los cambios de códigos (code switching),

- la sobreproducción (overproduction) o la producción reducida (underproduction),

- las dificultades al recordar cierta palabra (lexical retrieval problems),

- el tiempo de reacción más lento (slowdown in reaction time),

- la evitación del idioma (language avoidance),

- el desgaste del idioma (language attrition),

- la pérdida del idioma (language loss).

${ }^{1}$ «Transfer is the influence resulting from similarities and differences between the target language and any other language that has been previously (and perhaps imperfectly) acquired» (Odlin, 1989: 27). 
Tal como aparece en la bibliografía al respecto (Poplack, 2000, Grosjean, 1994, etc.), para designar el idioma que influye durante un cierto tiempo en el idioma de destino, se emplean los términos donor language, guest language, embedded language y non-base language, mientras que, para designar el idioma de base, se utilizan los de recipient language, host language, matrix language, o base language.

Los autores se fijan frecuentemente en la transferencia lingüística negativa, llamada también interferencia, bastante fácil de observar en los errores lingüísticos o en equivocaciones (Corder, 1967/1981), o también en errores de competencia (James 1998; Ecke, Hall, 2003). Paradis (2004: 188) y Grosjean (1994) distinguen, además, entre interferencia dinámica, que consiste en el uso único, en un determinado momento, de un elemento perteneciente a un idioma distinto al idioma básico, e interferencia estática, más arraigada, que consiste en el uso, durante el periodo de aprendizaje de un idioma, de un elemento que está almacenado en dos sistemas intralingüísticos.

\section{PERFIL DEL GRUPO EXAMINADO}

El grupo de informantes que hemos elegido para la observación de los diferentes fenómenos relacionados con la transferencia lingüística está constituido por estudiantes de II y III cursos de estudios de grado y de I y II cursos de estudios de máster en Filología Hispánica. Las observaciones fueron hechas durante las clases de otra lengua romance (en este caso, el portugués).

Hacemos la observación concerniente a la mezcla de idiomas, es decir, de las interlenguas en los niveles: léxico, sintáctico, fonológico y pragmático. En la producción diaria de los estudiantes se constatan hechos como el paso de un código a otro, la presencia de voces de procedencia extranjera o las interferencias. La universidad, concretamente las clases prácticas de idiomas, es el contexto social en el que se produce la mezcla de idiomas. Hay que señalar que, según el programa de estudios, no se imparte portugués en primer curso, sino a partir de segundo. Cabe suponer entonces que los estudiantes, al entrar en contacto por primera vez con el portugués, ya tienen como recursos, las prácticas, hábitos y estrategias del aprendizaje de idiomas extranjeros, obtenidos mediante sus experiencias profesionales. Tienen además una motivación que es, en gran medida, resultado de sus rasgos individuales internos y externos. Como se puede ver, estamos hablando de un ambiente artificial, dirigido, que sirve para aprender el idioma extranjero. En tales circunstancias puede prevalecer el interés por la forma sobre el interés por la funcionalidad comunicativa y emocional del enunciado. Las reglas del método comunicativo aplicadas durante las clases pueden únicamente "suavizar" los intentos de los estudiantes para tratar el idioma a través del prisma de su forma, lo que a la postre resulta solamente un sucedáneo de "una inmersión lingüística”. 
Hay que tener en cuenta que el español, además de en el aula, se aprende de modo natural de otras formas, como por ejemplo, en la conversación con hablantes nativos durante una estancia becada en España. Se produce aquí una cierta continuidad en lo que se refiere a la familiarización con el idioma extranjero, cuando este es el segundo idioma. En el caso del portugués, esta situación raramente tiene lugar, ya que es muy escaso el contacto que los estudiantes tienen con los hablantes nativos de portugués, al ser menos frecuentes las estancias becadas en Portugal.

Es difícil fijar el nivel de conocimiento del idioma L1 que determina la existencia de las influencias intralingüísticas. Seguramente influyen la etapa en que se inicia el aprendizaje del segundo idioma y la duración y frecuencia de uso del mismo. Las competencias y el grado de desarrollo son diferentes para cada idioma. Raramente se alcanza una simetría perfecta en cuanto a conocimiento y uso entre los dos idiomas que conoce una persona.

En el caso del grupo examinado, el idioma español es la asignatura principal del programa de estos estudios, por lo que el tiempo de exposición a este idioma supera con creces el tiempo de contacto con el idioma portugués. En esta situación es casi imposible que los estudiantes que aprenden portugués alcancen un nivel de competencia similar al idioma español. Aparte de esto, los estudiantes aprenden el idioma español en contextos mucho más amplios: estudian literatura, historia y otras asignaturas universitarias en la lengua meta mientras que en las clases de portugués se fijan más en la parte práctica y funcional del idioma, ampliado solamente con elementos literarios o culturales.

\section{EJEMPLOS DE TRANSFERENCIA LINGÜÍSTICA: ESTUDIO DE CASO}

En el caso del grupo examinado, se puede considerar la transferencia como un cierto tipo de estrategia "de socorro" o de compensación. Los estudiantes que todavía no conocen el idioma básico en un grado notable se sirven del idioma que conocen mucho mejor. Este tipo de influencias lingüísticas son tratadas como una estrategia también por otros estudiosos (Kellerman, 1977; Gabryś-Barker, 2005). En tal caso la transferencia como estrategia en sí misma es un proceso positivo; son solo los efectos de su uso los que pueden ser positivos o negativos.

Durante las clases se pueden observar los siguientes fenómenos:

- el cambio de código o desplazamiento total del término portugués por el término español, es decir, cuando se usa la palabra que no proviene del idioma básico, p. ej.:

1) Eu tenho um perro. (en lugar del portugués cão)

2) Eu queria viajar, pero não tenho dinheiro. (en lugar del portugués mas)

- cruces lingüísticos - híbridos - de lexemas procedentes del idioma básico con los morfemas del idioma L1, o al revés. 
3) Quedou em casa (esp. quedar en vez de port. ficar, en la unión con el morfema de la 3. ${ }^{\text {a }}$ persona del singular del pretérito perfecto simple).

Se aprecian también otros fenómenos de transferencia léxica, tales como:

— los cognados totales o parciales (falsos amigos)

Oficina -esp. biuro; port. warsztat (en vez de port. escritório)

— la copia de unidades complejas (ing. multiwords)

4) *Tenho ganas de sair à noite. (en vez de tenho vontade de...)

Los ejemplos presentados se concentran en la parte léxica del idioma. Sin embargo, las operaciones intralingüísticas que observamos en las clases conciernen también a otros aspectos del idioma, como la transferencia morfológica, que ocurre, por ejemplo, por la influencia de las reglas morfosintácticas del idioma base en la producción de la lengua meta.

Hay que tener en cuenta que los procesos mencionados entran en juego en las interacciones, siendo difícil asignar una determinada operación a un tipo concreto de transferencia. Pueden ocurrir además influencias entre todos los idiomas que conoce una persona multilingüe, incluido el idioma materno.

\section{FACTORES QUE INFLUYEN EN LAS INTERACCIONES LINGÜÍSTICAS}

Observando con atención la situación del grupo examinado, es fácil percatarse de que, durante el proceso de activación de un segundo idioma extranjero, el estudiante posee ya varios sistemas lingüísticos que se influencian recíprocamente. En este caso, son factores importantes el grado de parentesco de los idiomas, el orden de adquisición, el nivel de aprendizaje y el grado de competencia comunicativa alcanzado, así como las funciones y situaciones comunicativas en las que usa cada idioma en su vida. Es más, la producción y comprensión de una unidad léxica implican no solo la interacción entre dos o más sistemas lingüísticos, sino más bien entre las formas intralingüísticas, ya que cada uno de los idiomas asimilados por el estudiante tiene su forma intralingüística de evaluación, enteramente instrumental, que se encuentra en una determinada etapa de desarrollo. Entendido de esta manera, cada uno de los idiomas existentes en la mente del hablante multilingüe, incluyendo el idioma materno, tiene una forma intralingüística, de modo que todas estas formas se influencian recíprocamente.

Los factores que de una forma importante afectan a las influencias lingüísticas que se hacen evidentes durante las clases de portugués son:

- el grado de semejanza entre los idiomas: el que percibe el estudiante y el que en realidad existe;

- el nivel de competencia en cada uno de estos idiomas: es lógico que el idioma que conocemos mejor influya decisivamente en el idioma básico, aunque es preciso 
matizar esta afirmación. Si bien es cierto que el idioma mejor aprendido por los estudiantes es su idioma materno, no lo es menos que a menudo su influencia es limitada cuando predomina el criterio de la semejanza tipológica. No ponen en duda esta suposición los exámenes llevados a cabo por Muñoz (2007) en un grupo de jóvenes hispanohablantes o catalanohablantes que demuestran el aprovechamiento del idioma o los idiomas dominantes (español y catalán) en la enseñanza del inglés, aunque el idioma L2 de este grupo de estudiantes era el francés. Sin embargo, si el idioma L2 fuera otro idioma de la familia de las lenguas germánicas, quizá los resultados serían diferentes. Otra observación que resulta de los exámenes de Muñoz es que el número de influencias disminuye a la vez que mejora el nivel de competencia del idioma básico. La misma relación ha sido indicada también por otros estudiosos (Sikogukira, 1993; Bouvy, 2000; Hammemberg, 2001). Sin embargo, nuestras observaciones confirman que, aunque la competencia lingüística es un factor importante, lo es más la semejanza lingüística o intralingüística, como también ha demostrado De Angelis (2005: 401):

It cannot be assumed a priori that whenever two languages typologically close to each other are present in the speaker's mind and one of them is the speaker's native language, it is the native language that will have the most dominant role.

No es verdad que solo el idioma más fuerte — el mejor asimilado — pueda influir en el idioma más débil. Según la transferencia retroactiva, las influencias del idioma básico se hacen visibles en un idioma bastante asimilado, incluso en el propio idioma materno (Hammemberg, 1998-2009; Cenoz, 2003, Sercu, 2007).

La situación en la que se encuentran los estudiantes seguramente influye en la activación del idioma L1 (español). El estudio del portugués tiene lugar en el contexto de las clases universitarias, en la misma universidad, en el mismo ambiente, donde los estudiantes usan el español casi un $80 \%$ del tiempo. Además, en la elección del español como idioma de apoyo puede influir la conciencia de que el profesor conoce o, por lo menos, entiende los enunciados en este idioma. La influencia puede venir también del rango del español como el idioma dominante en el programa de estudios de esta facultad. En este punto, hay que recordar que la activación de un idioma como idioma de uso colectivo en el acto de la producción del idioma no básico es el resultado de muchos factores complejos. Un estudiante puede activar solo una zona, un cierto alcance semántico o un grupo de palabras funcionales (Poulisse, Bongaerts, 1994) a lo largo de la comunicación en L2.

Otro factor que ejerce influencia en las interacciones intralingüísticas es el modo en que los idiomas fueron adquiridos. Como sabemos, se pueden adquirir los idiomas de modo natural y artificial durante un proceso formal de aprendizaje. Se ha observado que se influyen entre sí los idiomas adquiridos del mismo modo (Vildomec, 1963; Singleton, 1987). David Singleton ha comprobado que los errores que aparecen en el idioma francés L5 son el resultado de las interacciones con los idiomas L4 español 
y L1 inglés. Todos han sido adquiridos de modo natural. El latín y el irlandés, que fueron aprendidos en el entorno escolar, no se han revelado de forma visible en el uso del idioma L5.

\section{ASPECTOS POSITIVOS DE LA TRANSFERENCIA LINGÜÍSTICA. EJEMPLOS DEL APROVECHAMIENTO DEL ERROR}

El grado de semejanza tipológica tiene una influencia importante en la velocidad del estudio y sus efectos. Se observa que, generalmente, algunos estudiantes con cierto dominio de español se familiarizan, asimilan y memorizan más rápidamente los elementos estructurales del portugués, al ser un idioma emparentado genética y tipológicamente con el castellano. El hecho de que el estudiante cometa errores provenientes del español significa que transforma el conocimiento lingüístico de forma cognitiva, consciente, aprovechando el conocimiento almacenado en la memoria de larga duración (declarativa) y no la de procedimiento (mecánica).

Conscientes de las semejanzas existentes, somos capaces de prever de algún modo los aspectos más problemáticos que pueden constituirse en fuente de posibles errores de naturaleza morfosintáctica.

El estudiante concentrado en la forma y la estructura del idioma presta más atención a los elementos de la temporalidad verbal. Así, es alta la probabilidad de usar el verbo haver para la estructura de los tiempos portugueses compuestos. Lo bueno es que los estudiantes ya tienen entre sus recursos lingüísticos el concepto de tiempo compuesto, y una idea clara de los elementos que lo forman y el alcance de su uso. Deben ser conscientes de que a veces es necesario cambiar un simple elemento para que sea correcta una construcción completa, incluso en el idioma básico. A este respecto, un análisis inicial de una selección de ejemplos limitará rápidamente la aparición de errores del tipo:

5) *Quando chegámos os amigos já haviam saído.

6) *Eu já havia visto esse filme.

Estas frases son una prueba de que los estudiantes aprovechan el conocimiento adquirido previamente, creando analogías, algo que debería tratarse como una etapa temporal en la adquisición del idioma y no como algo totalmente negativo, sino más bien como un elemento que constituye el punto de partida en el análisis del error y, en consecuencia, de su corrección.

Una situación parecida la podemos proporcionar en la aplicación del verbo estar respecto a la ubicación de los lugares (direcciones, edificios, ríos, etc.). El idioma español usa en tal situación el verbo estar. Se puede advertir previamente a los estudiantes que el portugués, a la hora de aplicar la regla "temporal-fijo", es más consecuente aplicando el verbo ser para la ubicación de las cosas que no cambian de lugar. Tras esta introducción, aparecerán con menos frecuencia las frases erróneas del tipo: 
7) * Onde está o supermercado?

8) *O lago está perto da minha casa.

Con el tiempo, tras sucesivas correcciones e instrucciones acerca de "la lógica del idioma portugués" estos errores desaparecerán totalmente.

En consecuencia, prever los contextos de semejanza lingüística y exponerlos de manera adecuada reduce el tiempo de activación de una cierta estructura, por lo que puede ser considerado como un elemento de ayuda en el aprendizaje del idioma L2.

\section{CONSIDERACIONES FINALES}

En la mente de una persona bilingüe o multilingüe no existen sistemas lingüísticos separados, perfectamente dominados. François Grosjean (1994) dice que una persona bilingüe no es la suma de dos personas monolingües. Nuestros estudiantes no son usuarios perfectos de dos idiomas, pero los usan con éxito en el nivel comunicativo, por eso, en nuestra opinión, es posible considerarlos bilingües en cuanto a conocimientos, competencias y destrezas, que, no obstante, difieren de una lengua a otra. Se puede hablar aquí de "multicompetencia», en tanto que una persona tiene en su mente dos o más sistemas lingüísticos relacionados entre sí, aunque no unidos, que se influencian recíprocamente. Se observa que, con la progresiva adquisición de una segunda lengua, la capacidad para "desactivar" una de ellas durante la producción es cada vez mayor o, dicho con otras palabras el grado de interferencia disminuye según aumentan las competencias en L2. Sin embargo, en los primeros estadios del aprendizaje de una nueva lengua, el estudiante se basa en lo ya existente, hace la reconstrucción y aprovecha los esquemas e ideas de pensamiento que ya posee para la asimilación de los nuevos, o adapta estos ya conocidos a los recién adquiridos, lo que significa una transformación intencional del material nuevo a base del conocimiento ya poseído. Las situaciones observadas en las clases demuestran que frecuentemente la mezcla de idiomas es el resultado de la elección consciente de los elementos, aquellos que, por la razón que sea, resultan más adecuados o más apropiados al hablante.

BIBLIOGRAFÍA

Apreciada Duarte, Cristina (1999): Diferencias de usos gramaticales entre español/portugués. Madrid: Editorial Edinumen.

Bouvy, Christine (2000): Towards the construction of the theory of cross-linguistics transfer. In: Jasone Cenoz, Ulrike Jessner, [eds.], English in Europe: The Acquisition of a Third Language. Clevedon: Multilingual Matters, 143-156.

CENOZ, Jasone (2001): The effect of linguistic distance, L2 status and age on cross-linguistic influence in third language acquisition. In: Jasone Cenoz, Britta HufeIsen, Ulrike Jessner [eds.], Crosslinguistic influence in third language acquisition: Psycholinguistic Perspectives. Clevedon: Multilingual Matters, 8-20. 
Cenoz, Jasone (2003): «The additive effect of bilingualism on third language acquisition». International Journal of Bilingualism, 7: 71-87.

Cenoz, Jasone, Britta Hufeisen, Ulrike Jessner [eds.] (2001): Cross-linguistic influence in third language acquisition: Psycholinguistic Perspectives. Clevedon: Multilingual Matters.

CORDER, Samuel (1967 [1981]): «The significance of learners' errors». International Review of Applied Linguistics, 5 (4): 161-169.

СнŁорек, Zofia (2011): Nabywanie języków trzecich i kolejnych oraz wielojęzyczność. Aspekty psycholingwistyczne (i inne). Wrocław: Wydawnictwo Uniwersytetu Wrocławskiego.

De Angelis, Gessica (2005): «Interlanguage Transfer of Function Words». Language Learning, 55: 379-414.

Gabryś-Barker, Danuta (2005): Aspects of Multilingual Storage, Processing and Retrival. Katowice, Wydawnictwo Uniwersytetu Śląskiego.

GrosJEan, François (1994): Individual bilingualism. In: Ron Asher [ed.], The Encyclopedia of Language and Linguistics. Oxford: Pergamon Press, 1656-1660.

Hall, Christopher, Ecke, Peter (2003): Parasitism as a default mechanism in L3 vocabulary acquisition. In: Jasone Cenoz, Britta HufeIsen, Ulrike Jessner [eds.], Cross-linguistic influence in third language acquisition: Psycholinguistic Perspectives. Clevedon: Multilingual Matters, 71-85.

Hammemberg, Björn (2001): Roles of L1 and L2 in L3 production and acquisition. Edinburgh: Edinburgh University Press.

Hammemberg, Björn [ed.] (2009): Processes in Third Language Acquisition. Edinburgh: Edinburgh University Press.

JAMES, Carl (1998): «Errors in Language Learning and Use: Exploring Error Analysis». Christopher N. CANDLIN [ed.], Applied Linguistics and Language Study, Longman Pearson Education.

Kellerman, Eric (1977): «Towards the characterization of the strategy of transfer in second language learning». Interlanguage Studies Bulletin, 2.1: 58-145.

MuÑoz, Carmen (2007): «Cross-linguistic influence and language switches in L4 oral production». Vigo International Journal of Applied Linguistics, 4: $73-94$ (special number) Current Issues in Second Language Acquisition.

OduIN, Terence (1989): Language transfer. Cross-linguistic influence in language learning. Cambridge: Cambridge University Press.

Paradis, Michel (2004): A Neurolinguistic Theory of Bilingualism. Amsterdam - Philadelphia, PA: John Benjamins.

Poplack, Shana (2000): «Sometimes I'll start a sentence in Spanish Y TERMINO EN ESPAÑOL, Towards a typology of code-switching». Linguistics, 18: 581-618.

Poulisse Nanda; BONGAERTS Theo (1994): «First language use in second language production». Applied Linguistics, 15 (1): 36-57.

Sercu, Lies (2007): «Acquiring multilingualism at school: What translation tasks tell us about adolescents' use of multilingual lexicon». International Journal of Multilingualism, 4: 52-75.

SiKogukiRA, Matutin (1993): «Influence of languages other than the L1 on a foreign language: a case of transfer from L2 to L3». Edinburgh Working Papers in Applied Linguistics, 4: $110-132$.

Singleton, David (1987): «Mother and other tongue influence on learner French: A case study». Studies in Second Language Acquisition, 9 (3): 327-345.

VILDOMEC, Vèroboj (1963): Multilingualism. Leyden: A.W. Sythoff. 